\title{
LES CANCERS DE L'ORBITE ETUDE RETROSPECTIVE À PROPOS DE 31 CAS
}

\author{
A. LACHKHEM, K. KHAMASSI, R. BEN HAMOUDA, S. TOUATI, H. FRIKHA*, \\ Z. OUESLATI, H. BOUSSEN ${ }^{* *}$, A. EL MAY***, S. GRITLI \\ SERVICE DE CHIRURGIE CARCINOLOGIQUE CERVICO-FACIALE ET ORL \\ * SERVICE DE RADIOTHÉRAPIE \\ ** SERVICE DE CARCINOLOGIE MÉDICALE \\ ***SERVICE D'IMMUNO-HISTO-CYTOLOGIE \\ INSTITUT SALAH AZAÏZ
}

\begin{abstract}
RESUME
Introduction : Les cancers de l'orbite sont rares. Ils sont caractérisés par leur diversité histologique et leur agressivité locorégionale rendant le traitement difficile et mutilant. Dans ce travail, nous rapportons notre expérience dans la prise en charge diagnostique et thérapeutique de ces tumeurs.

Matériels et méthodes : Notre étude rétrospective a concerné 31 cas de cancers de l'orbite colligés sur 13 ans (19932005). Tous les patients ont bénéficié d'un examen clinique complet, d'une imagerie du massif facial (TDM et/ou IRM) et d'une biopsie de la tumeur. Le traitement a été basé sur la chirurgie, la radiothérapie et/ou la chimiothérapie.

Résultats : La symptomatologie clinique était dominée par les signes ophtalmologiques et les algies faciales. L'imagerie a montré dans tous les cas un processus expansif tissulaire à point de départ orbitaire, avec lyse osseuse orbitaire chez 16 patients $(51,6 \%)$, une extension au massif facial chez 7 patients $(22,6 \%)$, endocrâniennes chez 6 patients (19,4\%) et des formes bilatérales atteignant les deux orbites dans 3 cas $(9,7 \%)$ L'anatomopathologie montrait une prédominance des lymphomes malins non hodgkiniens $(32,3 \%)$ et des carcinomes épidermoïdes $(32,3 \%)$, suivis des rhabdomyosarcomes embryonnaires (19,4\%).

Douze patients ont été traités par chirurgie et radiothérapie postopératoire, dix patients par une association radio-chimiothérapie, et neuf autres par une chimiothérapie néo-adjuvante. La survie globale était de $67,8 \%$ à 3 ans, $48,4 \%$ à 5 ans et $22,6 \%$ à 10 ans.

Conclusion : Les cancers de l'orbite sont de mauvais pronostic. Un diagnostic précoce et un traitement radical et adapté au type histologique permet d'améliorer la survie et la qualité de vie chez les patients atteints de ces tumeurs.

Mots-clés : Orbite, cancer, lymphome, carcinome épidermoïde, rhabdomyosarcome
\end{abstract}

Introduction : Orbit cancers are rare. They are characterised by histological diversity and local and regional aggressiveness, making their treatment difficult and mutilating. In this study, we report our experience in diagnostic and therapeutic management of these tumors.

Materials and methods : Our retrospective study concerned 31 cases of orbit cancers over 13 years (1993-2005). All patients had had complete clinical examination, sinonasal imaging (CT and/or MRI) and biopsy of the tumor. Treatment was based on surgery, radiotherapy and/or chemotherapy.

Results : Ophthalmologic signs and facial pain were the most frequent symptoms. Imaging showed in all cases an expansive process starting from the orbit, with orbital bony lysis in 16 patients $(51,6 \%)$, sinonasal extension in 7 patients $(22,6 \%)$, endocranial extension in 6 patients $(19,4 \%)$ and bilateral forms involving the two orbits in 3 cases $(9,7 \%)$. Anatomopathology showed predominance of non-Hodgkin's lymphomas (32,3\%) and epidermoid carcinomas (32,3\%), followed by embryonic rhabdomyosarcoma $(19,4 \%)$.

Twelve patients were treated by surgery and postoperative radiotherapy, ten patients with radio-chemotherapy association, and nine others with neoadjuvant chemotherapy. Global survival was $67,8 \%$ at 3 years, $48,4 \%$ at 5 years and $22,6 \%$ at 10 years.

Conclusion : Orbit cancers have prognosis. An early diagnosis and a radical treatment which is adapted to the histological type allow improving survival and quality of life of the patients having these tumors.

Keywords : Orbit, cancer, lymphoma, epidermoid carcinoma, rhabdomyosarcoma

\section{INTRODUCTION}

Les cancers de l'orbite sont des tumeurs relativement rares. Ils sont caractérisés par leur diversité histologique, leur survenue aux âges extrêmes et leur expression cli- nique assez univoque. Les progrès de l'imagerie médicale ont permis d'améliorer considérablement le diagnostic et la prise en charge thérapeutique de ces tumeurs. Cette étude rétrospective s'est intéressée aux caractéris- 
tiques épidémiologiques, cliniques, thérapeutiques et évolutives de ces tumeurs en les comparant à celles de la littérature.

\section{MATÉRIEL ET MÉTHODES}

Nous avons réalisé une analyse rétrospective des dossiers de 31 patients colligés et traités dans notre service pour un cancer de l'orbite pendant une période de 13 ans (1993-2005). Tous les patients ont bénéficié d'un examen clinique complet, d'une imagerie du massif facial (TDM et/ou IRM) et d'une biopsie de la tumeur. Le traitement a été basé sur la chirurgie, la radiothérapie (RT) et/ou la chimiothérapie (CT). La survie a été calculée selon la méthode de Kaplan-Meier. La surveillance a été clinique avec un recul moyen de 7 ans (15 mois-12 ans).

\section{RESULTATS}

L'âge moyen était de 44 ans (1-77 ans) et le sex-ratio 1,58 (19 hommes/12 femmes). Notre série comportait 6 enfants. Un patient avait des antécédents de carcinome épidermoïde de la lèvre inférieure traité par chirurgie et radiothérapie 7 ans auparavant.

La durée moyenne d'évolution de la symptomatologie était de 5 mois (3 mois-1 an) Les signes ophtalmologiques étaient les plus fréquents (100\%), suivis des signes neurologiques à type de douleurs orbitaires ou céphalées $(48,4 \%)$, et des signes rhinologiques (29\%) (Tableau I). L'examen ORL et ophtalmologique a trouvé une exophtalmie et/ou une tuméfaction orbitaire dans 25 cas $(80,6 \%)$, une baisse de l'acuité visuelle dans 15 cas $(48,4 \%)$, une ophtalmoplégie dans 8 cas $(25,8 \%)$ et des adénopathies sous digastriques dans 2 cas $(6 \%)$.

La TDM a été pratiquée chez 25 patients et l'IRM chez 8 patients. Elles ont objectivé un processus expansif tissulaire à point de départ orbitaire Une lyse osseuse orbitaire a été notée chez 16 patients $(51,6 \%)$ et une extension au massif facial chez 7 patients (22,6\%). L'extension endocrânienne a été notée dans 6 cas $(19,4 \%)$ et l'atteinte des deux orbites dans 3 cas $(9,7 \%)$ (Fig 1,2).

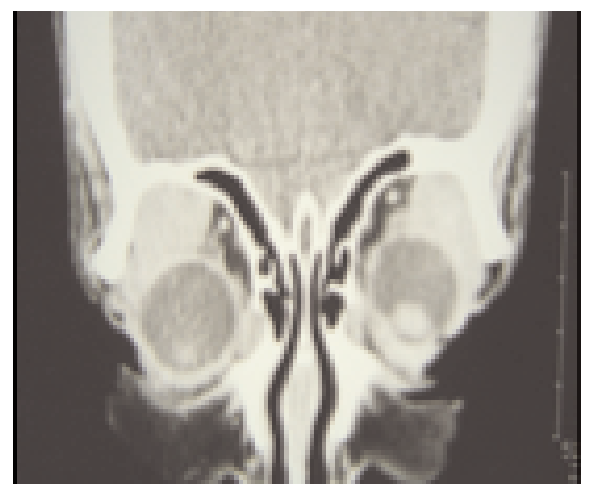

Fig 1: TDM (coupe coronale): Processus tissulaire intra orbitaire bilatéral (lymphome)

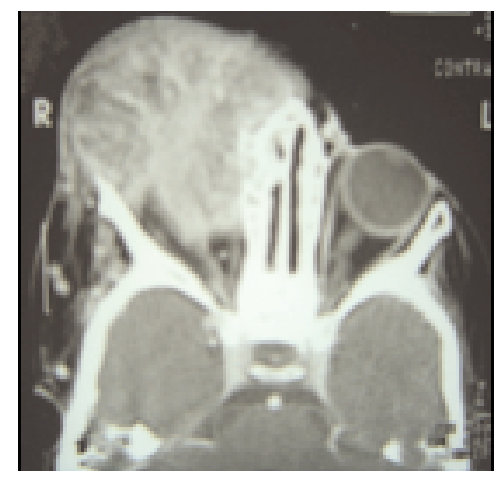

Fig 2: TDM (coupe axiale): Volumineux processus expansif tissulaire intra orbitaire droit (rhabdomyosarcome)

Une biopsie par voie externe trans-palpébrale a été pratiquée chez 27 patients et par voie endonasale chez 4 patients présentant une extériorisation nasale de la tumeur. L'anatomopathologie a conclu à la présence d'un lymphome malin non hodgkinien chez 10 patients $(32,3 \%)$ dont les 3 cas d'atteinte bilatérale à l'imagerie. Un carcinome épidermoïde a été noté chez 10 patients $(32,3 \%)$ et un rhabdomyosarcome embryonnaire chez 6 autres $(19,4 \%)$. Les autres types histologiques étaient plus rares (Tableau I).

\begin{tabular}{|l|l|c|c|}
\hline \multicolumn{2}{|l|}{} & Nombre de cas & Pour centages \\
\hline Symptomatologie & Signe s oculo-or bitai res & 31 & $100 \%$ \\
& exophtalmie+tuméfaction orbitaire & 25 & $80,6 \%$ \\
& baisse de l'acuité visuelle & 15 & $48,4 \%$ \\
& diplopie & 8 & $25,8 \%$ \\
& Algies faciales & 15 & $48,4 \%$ \\
& Signes rhin ologiq ues & 9 & $29 \%$ \\
\hline Extensions & Lyse osseuse orbitaire & 16 & $51,6 \%$ \\
& Massif facial & 7 & $22,6 \%$ \\
& Intracrânienne & 6 & $19,4 \%$ \\
& Formes bilatérales (lymphome) & 3 & $9,7 \%$ \\
\hline Anatomopathologie & Lymphome & 10 & $32,3 \%$ \\
& Carcinome épidermoïde & 10 & $32,3 \%$ \\
& Rhabdomyosarcome embryonnaire & 6 & $19,4 \%$ \\
& Adénocarcinome & 1 & $3,2 \%$ \\
& Mélanome malin & 1 & $3,2 \%$ \\
& Carcinome adénoïde kystique & 1 & $3,2 \%$ \\
& Liposarcome myxoïde & 1 & $3,2 \%$ \\
& Rétinoblastome & 1 & $3,2 \%$ \\
\hline
\end{tabular}

Tableau I: Tableau clinique, extensions et anatomopathologie

L'exentération orbitaire suivie de RT externe a été pratiquée chez 6 patients ayant un carcinome épidermoïde et chez les patients ayant un mélanome malin, un adénocarcinome lacrymal, un liposarcome myxoïde ou un carcinome adénoïde kystique.

Une exérèse chirurgicale conservatrice de globe oculaire suivie d'une RT externe a été pratiquée pour 2 enfants ayant un rhabdomyosarcome embryonnaire.

Une CT délivrant 3 cures en moyenne suivie de RT externe a été prescrite pour les 10 patients ayant un lympho- 
me. Une CT seule a été prescrite chez 4 patients ayant carcinome épidermoïde étendu jugé inopérable. Une CT seule a été également administrée à 4 enfants ayant un rhabdomyosarcome embryonnaire et à un patient ayant un rétinoblastome.

Sept patients $(22,6 \%)$ étaient décédés dans l'année qui a suivi le traitement. II s'agissait dans 5 cas d'une poursuite évolutive massive de la tumeur traitée symptomatiquement ( 2 cas de rhabdomyosarcome, 1 cas de carcinome épidermoïde, 1 cas d'adénocarcinome, 1 cas de carcinome adénoïde kystique). Les 2 autres décès étaient en rapport avec des métastases à distance (pulmonaires dans le cas du mélanome malin et osseuses dans le cas du liposarcome).

Sur les 31 patients, 10 étaient perdus de vue ou décédés à 3 ans $(32,3 \%), 16$ à 5 ans $(51,6 \%)$ et 24 à 10 ans $(77,4 \%)$. La survie globale, calculée selon la méthode de Kaplan-Meier, était de $67,8 \%$ à 3 ans, $48,4 \%$ à 5 ans et de $22,6 \%$ à 10 ans. L'étude de la survie en fonction du type histologique et du type de traitement est représentée dans le Tableau II.

\begin{tabular}{|c|c|c|c|}
\hline \multirow[b]{2}{*}{ Types histologiques et traitem ents } & \multicolumn{3}{|c|}{ Survie } \\
\hline & à 3 ans & à 5 ans & à 10 ans \\
\hline Carcinome épidermoïde & $7 / 10 \quad(70 \%)$ & $5 / 10(50 \%)$ & $3 / 10(30 \%)$ \\
\hline Lymphome & $6 / 10 \quad(60 \%)$ & $5 / 10(50 \%)$ & $2 / 10(20 \%)$ \\
\hline Rhabdomyosarcome embryonnaire & $3 / 6 \quad(50 \%)$ & $3 / 6 \quad(50 \%)$ & $1 / 6 \quad(16 \%)$ \\
\hline Chirurgie+RT & $9 / 12(75 \%)$ & $8 / 12(66.6 \%)$ & $2 / 12(16,6 \%)$ \\
\hline $\mathrm{CT} \pm \mathrm{RT}$ & $7 / 19(36,8 \%)$ & $5 / 19(26,3 \%)$ & $4 / 19(21 \%)$ \\
\hline
\end{tabular}

Tableau II: Pourcentages de survie en fonction du type histologique et du traitement (selon la méthode de Kaplan-Meier)

\section{DISCUSSION}

Les tumeurs malignes de l'orbite sont relativement rares (1). Elles sont caractérisées par leur diversité histologique selon la structure anatomique sur laquelle elles naissent. Elles sont le plus souvent primitives (mésenchymateuses, lymphoïdes, nerveuses, lacrymales, osseuses et vasculaires) et rarement secondaires à un cancer à distance $(2,3)$. Le lymphome malin non hodgkinien est décrit comme étant le type histologique le plus retrouvé dans nombreuses séries de la littérature, sa fréquence varie de 26 à $50 \%(1,2)$, ce qui concorde avec nos résultats $(32 \%)$. Les cancers des glandes lacrymales sont relativement rares, représentées surtout par les carcinomes (4). Parmi ceux-ci, on distingue les adénocarcinomes, les carcinomes mucoépidermoïdes, les carcinomes adénoïdes kystiques et les carcinomes sur adénome pléomorphe. Les carcinomes épidermoïdes sont surtout des tumeurs propagées à partir d'une tumeur des paupières ou des sinus de la face, ceux de la glande lacrymale sont rares
(4). Parmi les 10 carcinomes épidermoïdes de notre série, 7 avaient un point de départ palpébral et 3 naso-sinusien. Les tumeurs d'origine mésenchymateuse sont moins fréquentes, ils regroupent les sarcomes (fibrosarcomes, liposarcomes, rhabdomyosarcomes, ostéosarcomes) $(1,2)$. Les tumeurs d'origine neuro-ectodermique sont surtout représentées par les mélanomes malins. Les rétinoblastomes sont rares (2).

Ces tumeurs peuvent se rencontrer à tout âge, avec une prédilection des carcinomes et des lymphomes chez l'adulte et des rhabdomyosarcomes chez l'enfant (1). Le tableau clinique est assez stéréotypé, associant à des divers degrés des signes ophlalmologiques (exophtalmie, troubles de l'oculomotricité, baisse de l'acuité visuelle), des algies faciales et des signes inflammatoires. L'association à des signes rhinologiques traduit l'extension naso-sinusienne de la tumeur (1). L'examen ophtalmologique et $\mathrm{ORL}$ permet dans la majorité des cas de poser le diagnostic surtout en cas de syndrome tumoral. Dans les cas échéants, c'est l'imagerie qui permettra de visualiser la tumeur et de faire le bilan d'extension locorégional (4). A noter la possibilité de formes bilatérales rencontrés dans les lymphomes, le plus souvent de découverte radiologique (1). Dans notre série, l'atteinte bilatérale a été diagnostiquée radiologiquement chez 2 patients. Le diagnostic de malignité n'est confirmé qu'après une biopsie qui est faite par voie trans-palpébrale ou transconjonctivale, ou endonasale en cas d'extériorisation nasale de la tumeur (4).

La prise en charge thérapeutique de ces cancers est pluridisciplinaire incluant chirurgien, oncologue médical et radiothérapeute (4). En cas d'indication chirurgicale, la décision de procéder à une exentération orbitaire s'impose en cas d'atteinte du globe oculaire, des muscles oculomoteurs et / ou du nerf optique ou d'envahissement important de la périorbite (5). Ces atteintes sont évaluées préalablement sur l'imagerie, mais la décision finale de procéder à une exentération, notamment si la fonction visuelle est préservée, repose sur l'évaluation peropératoire de l'extension tumorale (6).

Les indications thérapeutiques dépendent essentiellement du type histologique, de l'extension tumorale et de l'âge du patient, tout en sachant qu'il s'agit d'un traitement lourd et mutilant. La chirurgie allant de la simple exérèse tumorale conservatrice à l'exentération orbitaire élargie aux tissus de voisinage et suivie de RT externe est préconisée pour les carcinomes et les rhabdomyosarcomes. Pour les carcinomes opérables, la préférence est à une chirurgie d'exérèse large complétée d'une RT externe (4). Pour les formes extensives jugées inopérables des carcinomes, l'association radio-chimiothérapie est indiquée 
(4). Pour les rhabdomyosarcomes, certains auteurs préconisent un traitement radical avec une exentération orbitaire large suivi de RT (7), d'autres préconisent une radiochimiothérapie qui permet d'obtenir un bon contrôle local de la tumeur mais avec un taux élevé de récidive $(7,8,9,10)$.

Le traitement de choix des lymphomes repose sur l'association radio-chimiothérapie (11). Certains auteurs préconisent une chirurgie d'exérèse ou une radiothérapie isolée dans les formes localisées (4). Pour les rétinoblastomes, l'association radio-chimiothérapie est préconisée (4).

Moyennant ces différentes indications, nous avons obtenu un taux de $77,4 \%$ de contrôle local de la maladie et un taux de survie de $48,4 \%$ à 5 ans.

La survie globale varie surtout en fonction du type histologique, de l'extension tumorale et des résultats du traitement. Ainsi la survie à 5 ans est de $75 \%$ pour les rhabdomyosarcomes (12,13), 64\% pour les lymphomes (11), et moins de $50 \%$ pour les carcinomes épidermoïdes et les carcinomes glandulaires (1), ce qui concorde avec les résultats de notre série.

\section{CONCLUSION}

Les cancers de l'orbite sont des tumeurs rares caractérisées par leur agressivité locorégionale, rendant leur traitement souvent difficile et mutilant et leur pronostic souvent médiocre tant sur le plan vital que sur le plan fonctionnel. Les progrès de l'imagerie médicale et des moyens thérapeutiques ont permis de contribuer à un diagnostic plus précoce et à une prise en charge thérapeutique adéquate permettant d'améliorer la survie et la qualité de vie chez les patients atteints de ces tumeurs.

\section{REFERENCES}

(1) Chadan N, Martin D, Iba-Zizen MT, Cabanio EA, Chailloux E. Processus expansifs intra orbitaires. A propos de 917 études rétrospectives d'un registre neuro-ophtalmologique de 6354 cas. Ophtalmologie 1992;6:39-42.

(2) Margo CE, Mulla ZD. Malignant tumours of the orbit. Analysis of the Florida cancer registry. Ophthalmology 1998;105:185-190.

(3) Shields JA, Shields CL, Scartozzi R. Survey of 1264 patients with orbital tumors and simulating lesions. Ophthalmology 2004;111: 997-1008.

(4) Ducasse A. Tumeurs de l'orbite de l'adulte. EMC (Elsevier, Paris), Ophtalmologie 21-650-A-20,1996,20p.

(5) Imola MJ, Schramm VL. Orbital preservation in surgical management of sinonasal malignancy. Laryngoscope 2002;112:1357-1365.

(6) Graamans K, Slootweg PJ. Orbital exenteration in surgery of malignant neoplasms of the paranasal sinuses. The value of preoperative computed tomography. Arch Otolaryngol Head Neck Surg 1989;115:977-980

(7) Le Gall F, Edan CH, Toulemont PJ, Jouan H, Urvoy M, Ramee MP. Rhabdomyosarcome orbitaire de l'enfant. A propos de 2 observations. J Fr Ophtalmol 1994;17:67-73.
(8) Offret G. Rhabdomyosarcome embryonnaire de l'enfant. A propos de 24 cas. Arch Ophthalmol 1976;36:709-732.

(9) Fiorillo A. Multidisciplinary treatment of primary orbital rhabdomyosarcoma. A single institution experience. Cancer 1991;67:560-563.

(10) Maurer HM. The intergroup rhabdomyosarcoma study II. Cancer 1993;71:1904-1922.

(11) Ahmed S, Shahid RK, Sison CP, Fuchs A, Mehrotra B. Orbital lymphomas: a clinocopathology study of a rare disease. Am J Med Sci 2006;331:79-83.

(12) Desjardins L. Les tumeurs en ophtalmo-pédiatrie. J Fr Ophtalmol 2000;23:926-939.

(13) Ducrey N, Nenadov-beck M, Spahn B. La thérapie actuelle du rhabdomyosarsome orbitaire de l'enfant. J Fr Ophtalmol 2002;25:298-302. 